\title{
The Influences of Withdrawal and Daily Dose Reduction of Pioglitazone on Metabolic Parameters in Patients With Type 2 Diabetes: A Retrospective Longitudinal Observational Study
}

\author{
Hisayuki Katsuyama ${ }^{a}$, Takayuki Fukunaga ${ }^{\mathrm{b}}$, Hidetaka Hamasaki ${ }^{\mathrm{a}}$, Hiroki Adachi ${ }^{\mathrm{a}}$, \\ Sumie Moriyama ${ }^{a}$, Akiko Kawaguchia ${ }^{a}$, Shuichi Mishima ${ }^{a}$, Akahito Sako ${ }^{\text {a }}$, Hidekatsu Yanai ${ }^{a}$ c
}

\begin{abstract}
Background: The aim of the study was to understand the influences of withdrawal or dose reduction of pioglitazone in patients with type 2 diabetes.

Methods: We retrospectively picked up patients who had undergone withdrawal or daily dose reduction of pioglitazone after a continuous prescription for 3 months or longer between January 2010 and March 2014. We compared the data before the withdrawal or dose reduction of pioglitazone with the data at 3 or 6 months after those by a chartbased analysis.
\end{abstract}

Results: Among 713 patients taking pioglitazone at least once during the studied period, 20 patients had undergone withdrawal of pioglitazone (group A) and 51 patients had undergone daily dose reduction (group B). The mean pioglitazone dose at baseline was $23 \mathrm{mg}$ in subjects of group A, and $30 \mathrm{mg}$ in group B. The number of subjects who had taken high-dose metformin $(\geq 1,000 \mathrm{mg}$ ) and dipeptidyl peptidase-4 (DPP-4) inhibitors increased after the withdrawal or dose reduction of pioglitazone in both groups. Although no significant change was observed in plasma glucose and $\mathrm{HbA} 1 \mathrm{c}$ levels, body weight significantly decreased at 3 and 6 months after the dose reduction in group B. The same tendency was observed in group A. Serum high-density lipoprotein-cholesterol (HDL-C) levels significantly decreased at 3 and 6 months after the withdrawal in group A. The serum alanine aminotransferase levels significantly increased 3 months after the withdrawal in group A.

Conclusions: Present study demonstrated that the withdrawal of pioglitazone exacerbated serum HDL-C and liver function in patients

Manuscript accepted for publication June 01, 2016

aDepartment of Internal Medicine, National Center for Global Health and Medicine Kohnodai Hospital, Chiba, Japan

${ }^{b}$ Department of Gastroenterology and Hepatology, National Center for Global Health and Medicine Kohnodai Hospital, Chiba, Japan

${ }^{\mathrm{c}}$ Corresponding Author: Hidekatsu Yanai, Department of Internal Medicine, National Center for Global Health and Medicine Kohnodai Hospital, 1-7-1 Kohnodai, Ichikawa, Chiba 272-0034, Japan. Email: dyanai@hospk.ncgm.go.jp

doi: http://dx.doi.org/10.14740/jocmr2611w with type 2 diabetes, although glycemic control could be maintained by using high-dose metformin or DPP-4 inhibitors.

Keywords: Body weight; HDL-cholesterol; Liver function; Pioglitazone; Withdrawal

\section{Introduction}

Pioglitazone is one of thiazolidinedione derivatives, which has been widely prescribed in patients with type 2 diabetes. It stimulates nuclear peroxisome proliferator-activated receptor gamma and improves insulin sensitivity by acting on adipose tissue, muscle and liver $[1,2]$. Previous reports showed that pioglitazone lowers plasma glucose levels and $\mathrm{HbAlc}$ when used as monotherapy or combination therapy with metformin, sulfonylurea and insulin [3]. Moreover, pioglitazone has been reported to improve cardiovascular risk factors including lipid profile, coronary atherosclerosis and carotid intima-media thickness [4-6].

Despite these beneficial effects, there are some concerns over pioglitazone to increase the risks of edema, body weight gain, congestive heart failure and bone fracture [7-9]. Furthermore, an epidemiological study by the French National Health Insurance showed that taking pioglitazone significantly increased risk of bladder cancer compared to patients who took other antidiabetic drugs [10]. The Food and Drug Administration also announced that pioglitazone use for more than 1 year might be associated with an increased risk of bladder cancer [11]. Because of these results, pioglitazone prescription was limited in many countries.

The Japanese authority also recommended to avoid prescribing pioglitazone to patients with a history of bladder cancer, and to monitor symptoms and urine in patients taking pioglitazone. As the news of possible association between pioglitazone and bladder cancer inflamed anxieties of diabetic patients, withdrawal or daily dose reduction of pioglitazone was done in a great number of patients. However, the influences of withdrawal or dose reduction have not been studied yet. Here, we retrospectively studied the influences of withdrawal or daily dose reduction of pioglitazone in patients with 
Table 1. Baseline Characteristics of the Study Subjects Who Had Undergone the Withdrawal of Pioglitazone $(n=20)$

\begin{tabular}{ll}
\hline Age & $68 \pm 12$ \\
Sex (male/female) & $13 / 7$ \\
Body height $(\mathrm{cm})$ & $157 \pm 10$ \\
Body weight $(\mathrm{kg})$ & $73.1 \pm 12.2$ \\
Body mass index $\left(\mathrm{kg} / \mathrm{m}^{2}\right)$ & $28.5 \pm 3.5$ \\
Plasma glucose $(\mathrm{mg} / \mathrm{dL})$ & $172 \pm 63$ \\
Hemoglobin Alc $(\%)$ & $6.9 \pm 0.9$ \\
Daily dose of pioglitazone $(\mathrm{mg})$ & $23 \pm 10$ \\
\hline
\end{tabular}

type 2 diabetes.

\section{Methods}

\section{Patients studied and data collection}

This study was approval by the Institutional Ethics Committee in National Center for Global Health and Medicine (NCGMG-001668), and was also performed in accordance with the Declaration of Helsinki. This study was registered with the University Hospital Medical Information Network (UMIN) clinical trials registry, number UMIN 000018104.

We selected patients with type 2 diabetes, who had undergone withdrawal or daily dose reduction of pioglitazone after a continuous prescription for 3 months or longer between January 2010 and March 2014, by using the clinical research database system at the National Center for Global Health and Medicine Kohnodai Hospital. We divided subjects into two groups: group A: patients who had undergone withdrawal of pioglitazone; group B: patients who had undergone dose reduction of pioglitazone.

We collected and compared the data before the withdrawal or dose reduction of pioglitazone with the data at 3 and 6 months after the withdrawal or dose reduction based on medical charts. Collected data include body weight, blood pressure, plasma glucose, HbA1c, serum low-density lipoprotein-cholesterol (LDL-C), triglyceride (TG), high-density
Table 2. Baseline Characteristics of the Study Subjects Who Had Undergone the Daily Dose Reduction of Pioglitazone (n $=51$ )

\begin{tabular}{ll}
\hline Age & $63 \pm 12$ \\
Sex (male/female) & $29 / 22$ \\
Body height $(\mathrm{cm})$ & $163 \pm 9$ \\
Body weight $(\mathrm{kg})$ & $72.5 \pm 20.5$ \\
Body mass index $\left(\mathrm{kg} / \mathrm{m}^{2}\right)$ & $27.7 \pm 5.8$ \\
Plasma glucose $(\mathrm{mg} / \mathrm{dL})$ & $163 \pm 66$ \\
Hemoglobin A1c $(\%)$ & $7.2 \pm 1.3$ \\
Daily dose of pioglitazone $(\mathrm{mg})$ & $30 \pm 10$ \\
\hline
\end{tabular}

lipoprotein-cholesterol (HDL-C), aspartate aminotransferase (AST), alanine aminotransferase (ALT) and creatinine which were measured almost at the same time point before, 3 and 6 months after the withdrawal or dose reduction of pioglitazone.

\section{Statistical analysis}

Comparison of the variables determined before and after was analyzed by a paired Student's $t$-test. All data were expressed as mean $\pm \mathrm{SD}$. $\mathrm{P}<0.05$ was considered to be statistically significant.

\section{Results}

We found 713 patients who had taken pioglitazone between January 2010 and March 2014. After a continuous prescription of pioglitazone for 3 months or longer, pioglitazone was withdrawn in 20 patients (group A), and the daily dose of pioglitazone was reduced in 51 patients (group B).

Tables 1 and 2 show the baseline characteristics of the studied subjects. The mean body mass index (BMI) was 28.5 $\mathrm{kg} / \mathrm{m}^{2}$ in group A and $27.7 \mathrm{~kg} / \mathrm{m}^{2}$ in group B. The mean pioglitazone dose at baseline was $22.5 \mathrm{mg}$ in subjects of group A. In subjects of group B, the mean dose of pioglitazone was 29.6 $\mathrm{mg}$ at baseline, $11.9 \mathrm{mg}$ at 3 months, and $11.7 \mathrm{mg}$ at 6 months

Table 3. Other Hypoglycemic or Lipid Modifying Agents Which Subjects Had Taken Before and After the Withdrawal of Pioglitazone $(n=20)$

\begin{tabular}{llll}
\hline Hypoglycemic and lipid modifying agents & Before withdrawal & $\mathbf{3}$ months after withdrawal & $\mathbf{6}$ months after withdrawal \\
\hline Metformin & $8(40 \%)$ & $9(45 \%)$ & $9(45 \%)$ \\
Metformin at high dose $(\geq 1,000 \mathrm{mg})$ & $0(0 \%)$ & $4(20 \%)$ & $4(20 \%)$ \\
Sulfonylureas & $3(15 \%)$ & $3(15 \%)$ & $5(25 \%)$ \\
Dipeptidyl peptidase-4 inhibitors & $1(5 \%)$ & $11(55 \%)$ & $12(60 \%)$ \\
$\alpha$-glucosidase inhibitors & $7(35 \%)$ & $6(30 \%)$ & $6(30 \%)$ \\
Glinides & $0(0 \%)$ & $0(0 \%)$ & $0(0 \%)$ \\
Insulin & $2(10 \%)$ & $2(10 \%)$ & $2(10 \%)$ \\
Statins & $6(30 \%)$ & $6(30 \%)$ & $6(30 \%)$ \\
Fibrates & $1(5 \%)$ & $0(0 \%)$ & $0(0 \%)$ \\
\hline
\end{tabular}


Table 4. Other Hypoglycemic or Lipid Modifying Agents Which Subjects Had Taken Before and After the Daily Dose Reduction of Pioglitazone $(n=51)$

\begin{tabular}{llll}
\hline Hypoglycemic and lipid modifying agents & Before reduction & $\mathbf{3}$ months after reduction & $\mathbf{6}$ months after reduction \\
\hline Metformin & $29(57 \%)$ & $32(63 \%)$ & $33(65 \%)$ \\
Metformin at high dose $(\geq 1,000 \mathrm{mg})$ & $15(29 \%)$ & $21(41 \%)$ & $25(49 \%)$ \\
Sulfonylureas & $15(29 \%)$ & $14(27 \%)$ & $14(27 \%)$ \\
Dipeptidyl peptidase-4 inhibitors & $19(37 \%)$ & $24(47 \%)$ & $24(47 \%)$ \\
$\alpha$-glucosidase inhibitors & $26(51 \%)$ & $24(47 \%)$ & $25(49 \%)$ \\
Glinides & $3(6 \%)$ & $6(12 \%)$ & $6(12 \%)$ \\
Insulin & $3(6 \%)$ & $3(6 \%)$ & $4(8 \%)$ \\
Statins & $26(51 \%)$ & $28(55 \%)$ & $29(57 \%)$ \\
Fibrates & $1(2 \%)$ & $1(2 \%)$ & $1(2 \%)$ \\
\hline
\end{tabular}

after the dose reduction.

Other hypoglycemic or lipid modifying agents which subjects had taken before and after the withdrawal or dose reduction of pioglitazone are shown in Tables 3 and 4. The number of subjects who had taken high-dose metformin $(\geq 1,000 \mathrm{mg})$ and dipeptidyl peptidase-4 (DPP-4) inhibitors increased after the withdrawal or dose reduction of pioglitazone in both groups.

Changes of clinical parameters after the withdrawal or daily dose reduction are shown in Tables 5 and 6 (group A) and Tables 7 and 8 (group B). Although no significant change was observed in plasma glucose and HbAlc levels in both groups, body weight significantly decreased at 3 and 6 months after the daily dose reduction in group B. The same tendency was observed in group A. Serum HDL-C levels significantly decreased at 3 and 6 months after the withdrawal in group A. Moreover, serum ALT levels significantly increased 3 months after the withdrawal of pioglitazone in group A. However, there were no significant changes in lipid profile and aminotransferases in group B.

\section{Discussion}

Present study evaluated the influences of withdrawal or daily dose reduction of pioglitazone, and demonstrated that serum HDL-C was exacerbated after the withdrawal, whereas plasma glucose and $\mathrm{HbA} 1 \mathrm{c}$ levels did not significantly change after the withdrawal or daily dose reduction.

In various studies, pioglitazone has been reported to improve not only glycemic control and insulin resistance, but also lipid profile [3, 4, 12]. Nevertheless, the number of reports about the influences of withdrawal of pioglitazone in patients is very limited. Iwase et al evaluated the effects of withdrawal of pioglitazone after 3-month treatment in 49 Japanese patients with type 2 diabetes, and revealed that plasma glucose, homeostatic model assessment insulin resistance (HOMA-IR), fasting serum TG and HDL-C returned to the levels before the pioglitazone treatment started [13]. In the retrospective cohort study, significant decreases in body weight and serum HDL$\mathrm{C}$ levels were observed after 10 months of the pioglitazone withdrawal [14]. These results were consistent with our data. No significant change was observed in serum TG levels in our study. Our study was based on the retrospective analysis and blood samples were taken in the post-prandial state in some patients, which might affect our results.

Several studies reported that administration of pioglitazone reduced steatosis and inflammation in liver in patients with non-alcoholic steatohepatitis (NASH) and type 2 diabetes $[15,16]$. Moreover, it was reported that stopping pioglitazone

Table 5. Changes of Clinical Parameters 3 Months After the Withdrawal of Pioglitazone

\begin{tabular}{lllll} 
& n & Before withdrawal & 3 months after withdrawal & P values \\
\hline Body weight (kg) & 6 & $72.2 \pm 13.8$ & $70.0 \pm 14.0$ & 0.193 \\
Plasma glucose (mg/dL) & 17 & $172 \pm 66$ & $142 \pm 49$ & 0.184 \\
Hemoglobin A1c (\%) & 14 & $6.7 \pm 0.9$ & $6.7 \pm 0.7$ & 0.790 \\
HDL-cholesterol (mg/dL) & 17 & $53 \pm 16$ & $48 \pm 16$ & 0.004 \\
LDL-cholesterol (mg/dL) & 14 & $91 \pm 26$ & $88 \pm 19$ & 0.608 \\
Triglyceride (mg/dL) & 18 & $166 \pm 123$ & $137 \pm 69$ & 0.336 \\
Aspartate aminotransferase (IU/L) & 18 & $22 \pm 8$ & $24 \pm 9$ & 0.193 \\
Alanine aminotransferase (IU/L) & 18 & $19 \pm 11$ & $24 \pm 14$ & 0.008 \\
Creatinine (mg/dL) & 18 & $0.86 \pm 0.21$ & $0.84 \pm 0.22$ & 0.663 \\
\hline
\end{tabular}


Table 6. Changes of Clinical Parameters 6 Months After the Withdrawal of Pioglitazone

\begin{tabular}{lllll}
\hline & n & Before withdrawal & 6 months after withdrawal & P values \\
\hline Body weight (kg) & 10 & $73.5 \pm 12.8$ & $71.4 \pm 12.4$ & 0.101 \\
Plasma glucose (mg/dL) & 17 & $172 \pm 66$ & $158 \pm 62$ & 0.421 \\
Hemoglobin A1c (\%) & 14 & $6.7 \pm 1.0$ & $6.7 \pm 0.8$ & 0.849 \\
HDL-cholesterol (mg/dL) & 17 & $53 \pm 16$ & $48 \pm 15$ & 0.006 \\
LDL-cholesterol (mg/dL) & 14 & $91 \pm 26$ & $86 \pm 20$ & 0.603 \\
Triglyceride (mg/dL) & 17 & $160 \pm 124$ & $133 \pm 71$ & 0.304 \\
Aspartate aminotransferase (IU/L) & 18 & $22 \pm 8$ & $23 \pm 9$ & 0.759 \\
Alanine aminotransferase (IU/L) & 18 & $19 \pm 11$ & $21 \pm 16$ & 0.318 \\
Creatinine (mg/dL) & 18 & $0.86 \pm 0.21$ & $0.84 \pm 0.20$ & 0.659 \\
\hline
\end{tabular}

Table 7. Changes of Clinical Parameters 3 Months After the Daily Dose Reduction of Pioglitazone

\begin{tabular}{lllll}
\hline & n & Before dose reduction & 3 months after dose reduction & P values \\
\hline Body weight (kg) & 34 & $75.0 \pm 2.10$ & $74.1 \pm 20.1$ & 0.030 \\
Plasma glucose (mg/dL) & 41 & $162 \pm 69$ & $150 \pm 57$ & 0.144 \\
Hemoglobin A1c (\%) & 35 & $7.2 \pm 1.3$ & $7.3 \pm 1.5$ & 0.245 \\
HDL-cholesterol (mg/dL) & 41 & $54 \pm 16$ & $55 \pm 16$ & 0.411 \\
LDL-cholesterol (mg/dL) & 26 & $99 \pm 21$ & $103 \pm 27$ & 0.427 \\
Triglyceride (mg/dL) & 41 & $155 \pm 141$ & $137 \pm 107$ & 0.217 \\
Aspartate aminotransferase (IU/L) & 42 & $27 \pm 18$ & $26 \pm 12$ & 0.716 \\
Alanine aminotransferase (IU/L) & 42 & $27 \pm 23$ & $26 \pm 18$ & 0.713 \\
Creatinine (mg/dL) & 41 & $0.76 \pm 0.25$ & $0.75 \pm 0.23$ & 0.480 \\
\hline
\end{tabular}

after 48-week treatment was associated with subsequent elevation in serum ALT levels, increase in total hepatic fat, and significant worsening of parenchymal inflammation and steatosis in patients with NASH [17]. A significant increase of serum ALT levels was also observed at 3 months after the withdrawal in present study. However, there was no significant change in serum ALT levels 6 months after the withdrawal. The reason was uncertain, but the limited number of subjects might be involved in the results.

Although no significant change was observed in plasma glucose and HbA1c levels in present study, the increase of plasma glucose levels after the withdrawal of pioglitazone was reported [13]. Morgan et al also reported that the glucose control worsened after the withdrawal of rosiglitazone, one of the thiazolidinediones [18]. In our study, the patients who had taken high-dose metformin or DPP-4 inhibitors increased. The intensification of treatment might contribute to keep their glycemic control.

In the previous study, 90 patients with type 2 diabetes were randomized to three groups: $7.5 \mathrm{mg} / \mathrm{day}, 15 \mathrm{mg} /$ day and

Table 8. Changes of Clinical Parameters 6 Months After the Daily Dose Reduction of Pioglitazone

\begin{tabular}{lllll}
\hline & n & Before dose reduction & 6 months after dose reduction & P values \\
\hline Body weight (kg) & 27 & $77.6 \pm 21.5$ & $76.3 \pm 20.9$ & 0.015 \\
Plasma glucose (mg/dL) & 39 & $161 \pm 71$ & $163 \pm 60$ & 0.823 \\
Hemoglobin A1c (\%) & 35 & $7.3 \pm 1.5$ & $7.4 \pm 1.3$ & 0.470 \\
HDL-cholesterol (mg/dL) & 39 & $54 \pm 16$ & $54 \pm 14$ & 0.744 \\
LDL-cholesterol (mg/dL) & 24 & $97 \pm 23$ & $101 \pm 23$ & 0.366 \\
Triglyceride (mg/dL) & 39 & $148 \pm 142$ & $149 \pm 128$ & 0.932 \\
Aspartate aminotransferase (IU/L) & 39 & $26 \pm 19$ & $24 \pm 7$ & 0.272 \\
Alanine aminotransferase (IU/L) & 38 & $27 \pm 23$ & $25 \pm 12$ & 0.457 \\
Creatinine (mg/dL) & 39 & $0.76 \pm 0.25$ & $0.76 \pm 0.25$ & 0.966 \\
\hline
\end{tabular}


$30 \mathrm{mg} /$ day as add-on therapy to metformin and/or sulfonylureas [19]. All three groups showed a significant reduction in HbA1c, C-peptide levels, HOMA-IR, TG and a significant increase in HDL-C [19]. The 15- and 30-mg pioglitazone groups showed a significant increase in body weight which was not seen in the low-dose $(7.5 \mathrm{mg} /$ day $)$ pioglitazone group, and there were no significant differences in any other parameters [19]. In our study, the pioglitazone dose reduction also reduced body weight, however, did not have an influence on any other metabolic parameters. Collectively, a low-dose pioglitazone may improve metabolic parameters without increasing body weight.

The present study has several limitations. First, other hypoglycemic agents, food intake and/or exercise might have an influence on the study results. Second, there were data lacking especially in BMI and body weight. Third, the number of studied subjects was small. Further studies, preferably with larger numbers of subjects, will be needed.

\section{Conclusion}

Our study suggested that the withdrawal of pioglitazone exacerbates serum lipid profile and liver function in patients with type 2 diabetes, although the glycemic control could be maintained by using high-dose metformin or DPP-4 inhibitors.

\section{Author Contributions}

HK and HY designed the research, collected and analyzed data, and wrote the paper. TF collected and analyzed data, and wrote the paper. $\mathrm{HH}, \mathrm{HA}, \mathrm{SM}, \mathrm{AK}, \mathrm{SM}$ and $\mathrm{AS}$ collected and analyzed data. All authors read and approved the final paper.

\section{Competing Interests}

The authors declare that they have no conflicts of interest concerning this article.

\section{Funding}

This work was supported in part by Grants-in-Aid for Research from the National Center for Global Health and Medicine (26-112).

\section{References}

1. Yki-Jarvinen $\mathrm{H}$. Thiazolidinediones. $\mathrm{N}$ Engl J Med. 2004;351(11):1106-1118.

2. Kadowaki T, Yamauchi T, Kubota N, Hara K, Ueki K, Tobe K. Adiponectin and adiponectin receptors in insulin resistance, diabetes, and the metabolic syndrome. J Clin Invest. 2006;116(7):1784-1792.

3. Kawamori R, Kadowaki T, Onji M, Seino Y, Akanuma
Y. Hepatic safety profile and glycemic control of pioglitazone in more than 20,000 patients with type 2 diabetes mellitus: postmarketing surveillance study in Japan. Diabetes Res Clin Pract. 2007;76(2):229-235.

4. Goldberg RB, Kendall DM, Deeg MA, Buse JB, Zagar AJ, Pinaire JA, Tan MH, et al. A comparison of lipid and glycemic effects of pioglitazone and rosiglitazone in patients with type 2 diabetes and dyslipidemia. Diabetes Care. 2005;28(7):1547-1554.

5. Nissen SE, Nicholls SJ, Wolski K, Nesto R, Kupfer S, Perez A, Jure $\mathrm{H}$, et al. Comparison of pioglitazone vs glimepiride on progression of coronary atherosclerosis in patients with type 2 diabetes: the PERISCOPE randomized controlled trial. JAMA. 2008;299(13):15611573 .

6. Mazzone T, Meyer PM, Feinstein SB, Davidson MH, Kondos GT, D'Agostino RB, Sr., Perez A, et al. Effect of pioglitazone compared with glimepiride on carotid intima-media thickness in type 2 diabetes: a randomized trial. JAMA. 2006;296(21):2572-2581.

7. Charbonnel BH, Matthews DR, Schernthaner G, Hanefeld M, Brunetti P. A long-term comparison of pioglitazone and gliclazide in patients with Type 2 diabetes mellitus: a randomized, double-blind, parallel-group comparison trial. Diabet Med. 2005;22(4):399-405.

8. Nesto RW, Bell D, Bonow RO, Fonseca V, Grundy SM, Horton ES, Le Winter M, et al. Thiazolidinedione use, fluid retention, and congestive heart failure: a consensus statement from the American Heart Association and American Diabetes Association. October 7, 2003. Circulation. 2003;108(23):2941-2948.

9. Loke YK, Singh S, Furberg CD. Long-term use of thiazolidinediones and fractures in type 2 diabetes: a metaanalysis. CMAJ. 2009;180(1):32-39.

10. Neumann A, Weill A, Ricordeau P, Fagot JP, Alla F, Allemand H. Pioglitazone and risk of bladder cancer among diabetic patients in France: a population-based cohort study. Diabetologia. 2012;55(7):1953-1962.

11. Lewis JD, Ferrara A, Peng T, Hedderson M, Bilker WB, Quesenberry CP, Jr., Vaughn DJ, et al. Risk of bladder cancer among diabetic patients treated with pioglitazone: interim report of a longitudinal cohort study. Diabetes Care. 2011;34(4):916-922.

12. Aronoff S, Rosenblatt S, Braithwaite S, Egan JW, Mathisen AL, Schneider RL. Pioglitazone hydrochloride monotherapy improves glycemic control in the treatment of patients with type 2 diabetes: a 6-month randomized placebo-controlled dose-response study. The Pioglitazone 001 Study Group. Diabetes Care. 2000;23(11):16051611.

13. Iwase M, Asano T, Sasaki N, Yoshizumi H, Hiramatsu S, Sakai Y, Ogo A, et al. Withdrawal of pioglitazone in patients with type 2 diabetes mellitus. J Clin Pharm Ther. 2010;35(4):401-408.

14. Lin KD, Lee MY, Feng CC, Chen BK, Yu ML, Shin SJ. Residual effect of reductions in red blood cell count and haematocrit and haemoglobin levels after 10-month withdrawal of pioglitazone in patients with Type 2 diabetes. Diabet Med. 2014;31(11):1341-1349. 
15. Belfort R, Harrison SA, Brown K, Darland C, Finch J, Hardies J, Balas B, et al. A placebo-controlled trial of pioglitazone in subjects with nonalcoholic steatohepatitis. N Engl J Med. 2006;355(22):2297-2307.

16. Teranishi T, Ohara T, Maeda K, Zenibayashi M, Kouyama K, Hirota Y, Kawamitsu H, et al. Effects of pioglitazone and metformin on intracellular lipid content in liver and skeletal muscle of individuals with type 2 diabetes mellitus. Metabolism. 2007;56(10):1418-1424.

17. Lutchman G, Modi A, Kleiner DE, Promrat K, Heller T, Ghany M, Borg B, et al. The effects of discontinuing pi- oglitazone in patients with nonalcoholic steatohepatitis. Hepatology. 2007;46(2):424-429.

18. Morgan CL, Puelles J, Poole CD, Currie CJ. The effect of withdrawal of rosiglitazone on treatment pathways, diabetes control and patient outcomes: a retrospective cohort study. J Diabetes Complications. 2014;28(3):360-364.

19. Rajagopalan S, Dutta P, Hota D, Bhansali A, Srinivasan A, Chakrabarti A. Effect of low dose pioglitazone on glycemic control and insulin resistance in Type 2 diabetes: A randomized, double blind, clinical trial. Diabetes Res Clin Pract. 2015;109(3):e32-35. 